\title{
Angiographic demonstration of intrahepatic termination of left gastric vein: a venous anomaly
}

\author{
Nicola H Strickland, P Dawson
}

Department of

Diagnostic Radiology,

Hammersmith Hospital,

London

Nicola H Strickland

$P$ Dawson

Correspondence to:

Dr Nicola Strickland, Dept of

Diagnostic Radiology,

Hammersmith Hospital,

W12 0HS.

Accepted for publication

23 July 1991
Du Cane Road, London

\begin{abstract}
An unusual venous anomaly is reported. A digital subtraction visceral angiogram shows intrahepatic termination of the left gastric (coronary) vein draining into branches of the left portal vein.
\end{abstract}

\section{Case report}

A 25 year old chef presented with a nine month history of recurrent attacks of severe epigastric

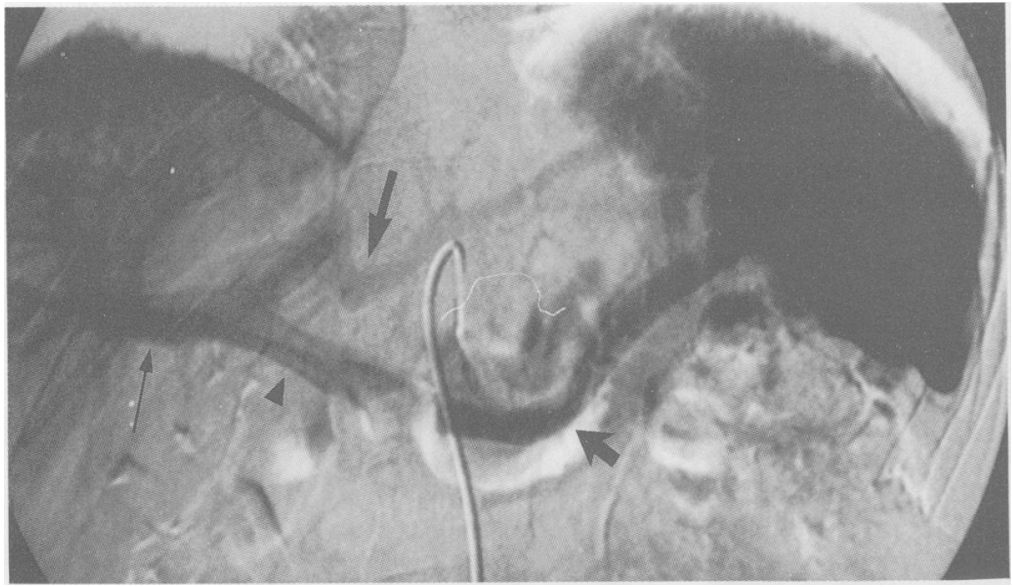

Figure 1: Indirect splenoportogram. Catheter tip in splenic artery. Left posterior oblique projection. ( ) splenic vein; ( $<$ ) main portal vein; ( - left portal vein; $(\longleftarrow)$ right portal vein.

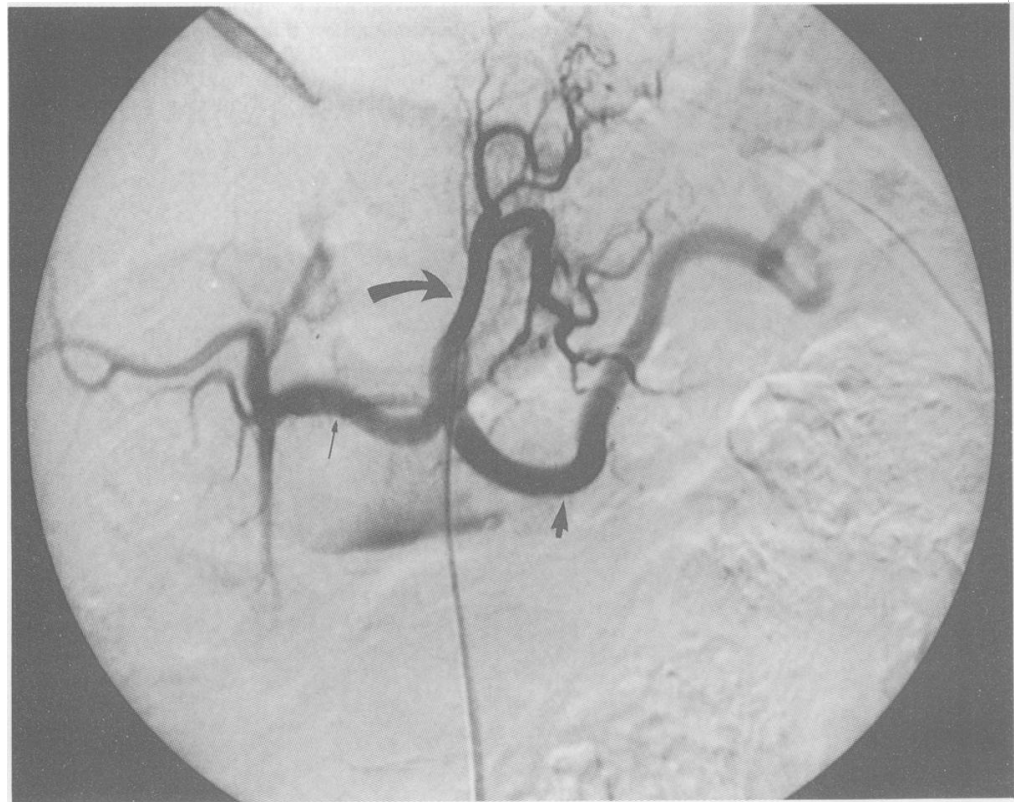

Figure 2: Arterial phase of coeliac axis angiogram. $(\rightarrow)$ splenic artery; $(\rightarrow)$ hepatic artery; $(\longrightarrow$ ) large left gastric artery. pain radiating through to the back. He had consumed half a bottle of whisky a day for the previous seven years. Examination revealed epigastric tenderness but no abnormal physical signs. In particular there was no evidence of portal hypertension. Routine haematological and biochemical investigations were normal, as were exocrine and endocrine pancreatic function tests. A diagnosis of chronic alcoholic pancreatitis was made based on the history and a computed tomography scan showing a very enlarged head of pancreas with a small pseudocyst, although no calcification was present.

Routine visceral angiography was carried out to provide a 'road map' of the vascular anatomy before surgical resection of the diseased pancreatic head. This was normal apart from an unusual anatomical variant illustrated in this report: a large left gastric vein was present which drained intrahepatically into terminal radicals of the left portal vein in the lateral segment of the left lobe of the liver, instead of following its conventional course draining into the main portal vein at the upper border of the first part of the duodenum. The gastric artery was also large.

\section{Discussion}

Only four definite cases of this interesting venous anomaly have been reported in the literature, all post mortem. During anatomical dissection, Kubik and Groscurth ${ }^{2}$ found such an enlarged left gastric vein in association with an accessory bile duct. The latter arose from the superolateral segment of the liver and drained into the gastric pylorus. Three other cadaveric demonstrations of intrahepatic termination of the left gastric vein are documented. ${ }^{3+}$ Douglass et $a l^{5}$ identified the left gastric vein in 90 cadaveric specimens and believed that in two of these it may have terminated within the liver as it could not be identified entering the main portal system (but insufficient hepatic tissue remained to permit investigation of the intrahepatic portal vein). None of these cases was associated with portal hypertenson.

We believe our case to be the first documentation of the in vivo angiographic demonstration of an aberrant course of the left gastric (coronary/ cardinal) vein joining intrahepatic branches of the left portal vein.

It is only with the relatively recent introduction of digital subtraction angiography that the venous phase of visceral angiograms has been routinely recorded with the high contrast resolution inherent to this imaging technique. Therefore it could be that this venous variant is in fact more common than would appear from the published literature. 

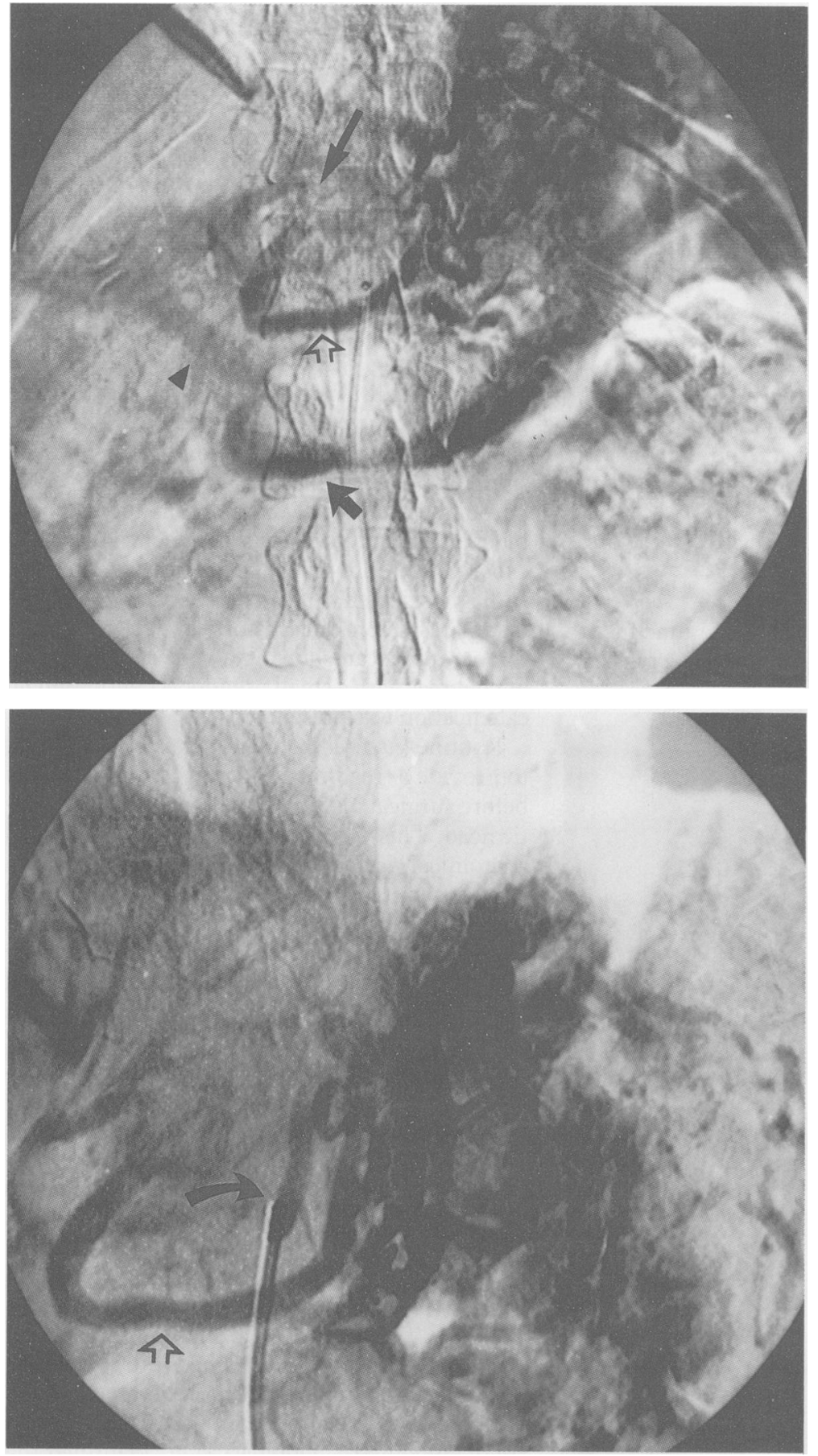

Figure 3: Venous phase of coeliac axis angiogram. Anteroposterior view. ( \& aberant left gastric vein draining into branches of left portal vein $(\longleftarrow)$ ); ( $<$ ) splenic vein; (4) main portal vein.

Figure 4: Magnified view of aberant left gastric vein $\leftarrow$ ) draining into left portal vein branches. $(\longrightarrow$ ) catheter tip selectively in left gastric artery, which is opacified by a small amount of contrast.

1 Johnston TB, Whillis J, eds. Gray's Anatomy. 29 ed. Longmans, Green, 1946: 862.

2 Kubik S, Groscurth P. Rare abnormality of extrahepatic bile duct and the V. coronaria ventriculi. Chirurg 1971; 48: 77-80.

3 Nebot-Cegarra J, Domenech-Mateu JM. Intrahepatic termination of the left gastric vein (vena gastrica sinistra): a new case of this unusual anatomic variation. Anat Anz 1986; 161: 309 15

4 Miyaki T, Yamada M, Kumaki K. Aberrant course of the lef gastric vein in the human. Possibility of a persistent left porta vein. Acta Anat (Basel) 1987; 130: 275-9.

5 Douglass BE, Baggenstoss AH, Hollinshead WH. The anatomy of the portal vein and its tributaries. Surg Gynecol Obstet 1950 91: 562-76. 